\title{
Stability Study Data File Reason Terminology
}

National Cancer Institute

\section{Source}

National Cancer Institute. Stability Study Data File Reason Terminology. NCI Thesaurus.

Code C96071.

Terminology developed to support Data File Reasons within the Stability Data Standards. 